\title{
OPERATOR COMMUTATIVITY IN JORDAN ALGEBRAS
}

\author{
N. JACOBSON ${ }^{1}$
}

If $a$ and $b$ are elements of a Jordan algebra $\mathfrak{A}$ we say that $a$ and $b$ operator-commute or o-commute if the multiplications $R_{a}$ and $R_{b}$ commute. Here $R_{a}$ is the linear transformation $x \rightarrow x a=a x$ of $\mathfrak{A}$. The notion of $o$-commutativity has been introduced by Jordan, Wigner, and von Neumann [4] who called this concept simply commutativity. Since every Jordan algebra is commutative in the usual sense, the above change in terminology seems to be desirable. In this note we shall study the notion of $o$-commutativity for finite-dimensional Jordan algebras of characteristic 0 . Our results are based on those of two previous papers $[1 ; 2]$.

1. If $\mathfrak{B}$ is a subset of the Jordan algebra $\mathfrak{A}$, then we denote by $\mathfrak{E} \mathfrak{\mathfrak { a }}(\mathfrak{B})$ the subset of $\mathfrak{A}$ of elements which $\boldsymbol{o}$-commute with every $b \in \mathfrak{B}$. Evidently $\mathfrak{E}_{\mathfrak{x}}(\mathfrak{B})$ is a subspace of $\mathfrak{A}$, but, as we shall see presently, it is not always a subalgebra. Assume now that $\mathfrak{A}$ is a special Jordan algebra, that is, $\mathfrak{A}$ is a subspace of an associative algebra $\mathfrak{U}$ closed relative to the Jordan multiplication $\{a b\}=a b+b a$ where $a b$ now denotes the associative multiplication. The condition $\left[R_{a} R_{b}\right]=0$ now gives $\{\{a x\} b\}=\{a\{x b\}\}$ for all $x$. This is readily seen to be equivalent to $[[a b] x]=0$ where $[a b]=a b-b a$. Hence we have the lemma:

LEMMA. If $\mathfrak{A}$ is a special Jordan algebra, $a$ and $b$ o-commute if and only if $[a b]$ is in the center of the enveloping associative algebra $\mathfrak{A}^{*}$ of $\mathfrak{A}$.

We can now construct an example in which $\mathfrak{E}_{\mathfrak{x}}(\mathfrak{B})$ is not an algebra. Let $a, b, c$ be finite matrices such that

$$
[a b]=c, \quad[a c]=0=[b c], \quad c^{2} \neq 0 .
$$

Such matrices have been constructed by McCoy [5]. Let $\mathfrak{A}$ be the enveloping Jordan algebra. Then $b \in \mathfrak{E}_{\mathfrak{x}}(a)$ but $\left[a, b^{2}\right]=2 b c$ and $\left[\left[a b^{2}\right] a\right]=2 c^{2} \neq 0$. Hence $b^{2} \in \mathfrak{E}_{\mathfrak{A}}(a)$. We remark also that $a$ and $b$ do not $o$-commute in the Jordan algebra of all the matrices.

In this note we shall consider $\mathfrak{E}_{\mathfrak{X}}(\mathfrak{B})$ such that either $\mathfrak{A}$ or $\mathfrak{B}$ is semisimple. Our first result is as follows:

THEOREM 1. Let $\mathfrak{A}$ be a special semi-simple finite-dimensional Jordan

Received by the editors March 3, 1952.

1 John Simon Guggenheim Memorial Fellow. 
algebra of characteristic 0 . Then $\mathfrak{C}_{\mathfrak{X}}(\mathfrak{B})$ is a subalgebra for any subset $\mathfrak{B}$ of $\mathfrak{A}$.

Proof. We may assume that $\mathfrak{A}$ is an algebra of linear transformations in a finite-dimensional vector space. It is known that $\mathfrak{A}$ is then completely reducible ([3] or [2]). If $a$ and $b o$-commute, $[a b]$ is in the center. Hence if $\mathfrak{R}$ denotes the enveloping Lie algebra of $\mathfrak{A}$, then $[a b] \in \mathbb{R}^{\prime} \cap \mathfrak{E}, \mathbb{R}^{\prime}$ the derived algebra and $\mathfrak{S}$ the center of $\mathfrak{R}^{\prime}$. Since $\mathfrak{R}$ is completely reducible, $\mathfrak{R}^{\prime} \cap \mathfrak{S}=0[1]$. Hence $[a b]=0$. Thus 0 -commutativity in $\mathfrak{A}$ is equivalent to commutativity. The conclusion is now clear.

2. We suppose next that $\mathfrak{B}$ is any finite-dimensional semi-simple Jordan algebra of characteristic 0 . The containing algebra $\mathfrak{A}$ can be arbitrary (even infinite-dimensional). We shall show that in this case also $\mathfrak{V}_{\mathfrak{X}}(\mathfrak{B})$ is a subalgebra.

Lemma 1. If $e$ is an idempotent element, then $\mathfrak{C}_{\mathfrak{A}}(e)$ is a subalgebra. ${ }^{2}$

Proof. Write $\mathfrak{A}=\mathfrak{A}_{0} \oplus \mathfrak{A}_{1 / 2}+\mathfrak{P}_{1}$ where $\mathfrak{A}_{i}=\left\{x_{i} \mid\right.$ ex $\left.x_{i}=i x_{i}\right\}, i=0$, $1 / 2,1$. If $u \in \mathfrak{A}_{0}, e u=0$. Since

$$
\left[R_{a b} R_{c}\right]+\left[R_{b c} R_{a}\right]+\left[R_{a c} R_{b}\right]=0
$$

substitution of $a=u, b=c=e$ gives $\left[R_{e} R_{u}\right]=0$. Hence $\mathfrak{A}_{0} \subseteq \mathfrak{F}_{\mathfrak{I}}(e)$. Similarly $\mathfrak{A}_{1} \subseteq \mathfrak{V}_{\mathfrak{I}}(e)$. On the other hand, if $v \in \mathfrak{E}_{\mathfrak{A}}(e) \cap \mathfrak{A}_{1 / 2}$, then $e(e v)$ $=(e e) v=v$ so that $(1 / 4) v=(1 / 2) v$ and $v=0$. Hence $\mathfrak{E}_{\mathfrak{a}}(e)=\mathfrak{A}_{0}+\mathfrak{A}_{1}$. It is known that $\mathfrak{H}_{0}^{2}=\mathfrak{A}_{0}, \mathfrak{A}_{1}^{2}=\mathfrak{A}_{1}, \mathfrak{A}_{0} \mathfrak{A}_{1}=0$ [4]. Hence $\mathfrak{A}_{0}+\mathfrak{A}_{1}$ is a subalgebra.

LEMMA 2. Let $\mathfrak{A}$ be a semi-simple Jordan algebra of finite dimensions over an algebraically closed field of characteristic 0 . Then $\mathfrak{A}$ has a basis consisting of idempotent elements.

Proof. It suffices to show that every element in $\mathfrak{A}$ can be written as a linear combination of idempotent elements. Since $\mathfrak{A}$ is power associative, any $a \in \mathfrak{A}$ can be written in one and only one way as $a=s+n$ where $s$ and $n$ are polynomials in $a, n$ is nilpotent, and $s$ is "semisimple" in the sense that its minimum polynomial has distinct roots. We recall also that the minimum polynomial of $R_{8}$ has distinct roots and that $R_{n}$ is nilpotent [6]. Also the $R_{a} k$ commute. Hence $R_{8}$ and $R_{n}$ commute, so that $R_{a}=R_{8}+R_{n}$ is the usual decomposition of a linear transformation into its semi-simple and its nilpotent parts. If $n=0$, $a=s$ can be written as a linear combination of orthogonal idempotent

\footnotetext{
2 This lemma is due to von Neumann [7, p. 476]. The idea of reducing our discussion to this result is suggested by a procedure of von Neumann.
} 
elements belonging to the algebra generated by $a$. Also since $\mathfrak{A}$ has an identity, $n=0$ if and only if $R_{n}=0$. Hence we shall attempt to prove the lemma by induction on the degree of nilpotency of $R_{n}$. It is known that since $\mathfrak{A}$ is semi-simple, $\mathfrak{A}$ contains a second nilpotent element $p$ such that

$$
n^{2} p-n(n p)=n, \quad p^{2} n-p(p n)=p[1] .
$$

In general we have the relation

$$
\left[\left[R_{a} R_{b}\right] R_{c}\right]=R_{A(b, c, a)}
$$

where $A(b, c, a)=(b c) a-b(c a)$. Hence it follows from (3) that the space spanned by $R_{n}, R_{p}$ and $\left[R_{n} R_{p}\right]$ is a three-dimensional simple Lie algebra (cf. $[1$, p. 111]). The theory of representations of these algebras shows that we can take the matrices of $R_{n}$ and $R_{p}$ to have diagonal block form where the blocks of $R_{n}$ and $R_{p}$ look like

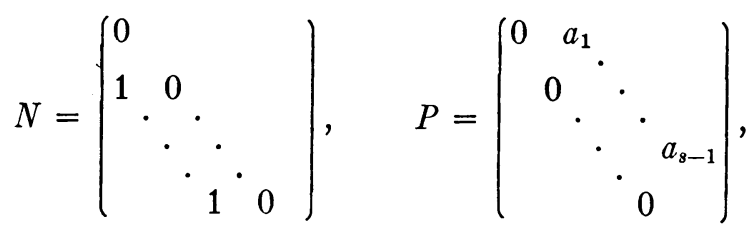

respectively, where the $a_{i}$ are negative rational numbers. The index of nilpotency of $R_{n}$ is the largest $s$ for these blocks. Now consider the element $n+p$. The matrix of $R_{n+p}$ has the same block form as $N$ and $P$ with $N+P$ replacing $N$ and $P$. A simple calculation shows that the characteristic polynomial of $N+P$ has the form $\lambda^{s}$ $-\left(\sum a_{i}\right) \lambda^{s-2}+\cdots$. Since $\sum a_{i} \neq 0$, this has at least two distinct roots. Hence we can decompose $N+P$ into smaller blocks. It follows that $R_{u}$ for the nilpotent part $u$ of $n+p$ has index $<$ index $R_{n}$. Hence we may suppose that $n+p$ is a linear combination of idempotents. The same reasoning applies to $n-p$. Hence $n$ is a linear combination of idempotents.

THEOREM 2. Let $\mathfrak{A}$ be an arbitrary Jordan algebra of characteristic 0 , $\mathfrak{B}$ a finite-dimensional semi-simple subalgebra. Then $\mathfrak{V}_{\mathfrak{x}}(\mathfrak{B})$ is a subalgebra.

Proof. The usual field extension argument shows that it suffices to prove the theorem for algebraically closed base fields. In this case we can find a basis $\left\{e_{1}, \cdots, e_{n}\right\}$ for $\mathfrak{B}$ of idempotents $e_{i}$. Then $\mathfrak{E}_{\mathfrak{X}}(\mathfrak{B})$ $=\cap \mathfrak{E}_{\mathfrak{a}}\left(e_{i}\right)$ and each $\mathcal{E}_{\mathfrak{q}}\left(e_{i}\right)$ is an algebra by Lemma 1 .

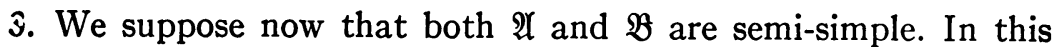
case we have the following result. 
Theorem 3. Let $\mathfrak{A}$ be a finite-dimensional semi-simple Jordan algebra of characteristic 0 and $\mathfrak{B}$ a semi-simple subalgebra. Then $\mathfrak{E}_{\mathfrak{x}}(\mathfrak{B})$ is a semi-simple algebra.

Proof. Write $\mathfrak{C}=\mathfrak{C}_{\mathfrak{r}}(\mathfrak{B})$ and set $R(\mathfrak{A})=\left\{R_{a} \mid a \in \mathfrak{A}\right\}$. Define $R(\mathfrak{B})$ and $R(\mathfrak{S})$ in a similar manner (as sets of multiplications acting in $\mathfrak{U}$ ). Then $\mathfrak{l}=R(\mathfrak{U})+[R(\mathfrak{U}), R(\mathfrak{U}))]$ is a completely reducible Lie algebra of linear transformations. The same is true of $\mathfrak{M}=R(\mathfrak{B})+[R(\mathfrak{B})$, $R(\mathfrak{B}))][2$, p. 526]). Hence the centralizer $\mathfrak{N}$ of $\mathfrak{M}$ in $\mathfrak{R}$ is completely reducible. Let $R_{u}+\sum\left[R_{v_{i}} R_{w_{i}}\right] \in \mathfrak{N}$. Then for $b \in \mathfrak{B}$

$$
0=\left[R_{u} R_{b}\right]+\sum\left[\left[R_{v_{i}} R_{w_{i}}\right] R_{b}\right]=\left[R_{u} R_{b}\right]+\sum R_{A\left(w_{i}, b, v_{i}\right)}
$$

where $A\left(w_{i}, b, v_{i}\right)=\left(w_{i} b\right) v_{i}-w_{i}\left(b v_{i}\right)$. Since $\mathfrak{A}$ has an identity, $R(\mathfrak{A})$ $\cap[R(\mathfrak{A}), R(\mathfrak{A})]=0$. Hence $\left[R_{u} R_{b}\right]=0$ and $\Sigma R_{A\left(w_{i}, b_{,} v_{i}\right)}=0$. Thus $u \in \mathbb{E}$ and $\Sigma\left[R_{v_{i}} R_{v_{i}}\right] \in \mathfrak{R}=\mathfrak{N} \cap[R(\mathfrak{H}), R(\mathfrak{A})]$. Evidently $\mathfrak{R}=R(\mathfrak{S}) \oplus \Re$ and we can verify that

$$
[R(\Im), \Omega] \subseteq R(\Im), \quad[\Omega \Re] \subseteq \Omega, \quad[R(\mathfrak{S}), R(\mathfrak{\Im})] \subseteq \Omega
$$

Let $u$ be a nilpotent element belonging to $\mathfrak{C}$. Then $R_{u}$ is nilpotent. The proof of Lemma 4 of [1] shows that there exists $R_{v}, v \in \mathbb{E}$, such that

$$
\left[\left[R_{v} R_{u}\right] R_{u}\right]=R_{u}, \quad\left[\left[R_{u} R_{v}\right] R_{v}\right]=R_{v} .
$$

This implies that

$$
v^{2} u-v(v u)=v, \quad u^{2} v-u(u v)=u
$$

and that $v$ is nilpotent. The existence of such a $v \in \mathbb{E}$ for every nilpotent $u \in \mathbb{S}$ implies that $\mathbb{S}$ is semi-simple [1].

\section{REFERENCES}

1. N. Jacobson, Completely reducible Lie algebras of linear transformations, Proceedings of the American Mathematical Society vol. 2 (1951) pp. 105-113.

2. - General representation theory of Jordan algebras, Trans. Amer. Math. Soc. vol. 70 (1951) pp. 509-530.

3. N. Jacobson and F. D. Jacobson, Classification and representation of semi-simple Jordan algebras, Trans. Amer. Math. Soc. vol. 65 (1949) pp. 141-169.

4. P. Jordan, J. v. Neumann, and E. Wigner, On an algebraic generalization of the quantum mechanical formalism, Ann. of Math. vol. 35 (1934) pp. 29-64.

5. W. H. Mills, $A$ theorem on the representation theory of Jordan algebras, Pacific Journal of Mathematics vol. 1 (1951) pp. 255-264.

6. N. H. McCoy, On quasi-commutative matrices, Trans. Amer. Math. Soc. vol. 36 (1934) pp. 327-340.

7. J. v. Neumann, On an algebraic generalization of the quantum mechanical formalism, Mat. Sbornik vol. 1 (1936) pp. 415-482.

YALE UNIVERSITY AND

UnIVERSITY OF PARIS 